\title{
Coverage-Enhancing algorithm for video sensor network based on improved particle swarm optimization
}

\author{
Xiang $\mathrm{Fu}^{1,}$, Jiexian Zeng ${ }^{2, b}$ \\ ${ }^{1}$ School of Software, Nanchang Hangkong University, Nanchang, 330063, China \\ ${ }^{2}$ School of Software, Nanchang Hangkong University, Nanchang, 330063, China \\ aemail: fxfb163@163.com, bemail: zengjx58@163.com
}

Keywords: Video sensor networks; Sensing coverage; Particle swarm optimization (PSO)

\begin{abstract}
The parameters of traditional particle swarm optimization (PSO) methods are unchangeable, which may lead to the iterations have slow convergence speed or unable to converge to global optimum. In this paper, improved PSO algorithm is applied to the coverage of video sensor network. Through improving inertia factors of PSO, the algorithm has great local search ability thus can avoid converging to local optimum value, and it can converge quickly to the global optimum value. Experiment results show that the proposed method has faster convergence speed and better coverage rate than traditional method based on PSO.
\end{abstract}

\section{Introduction}

Wireless sensor networks can be deployed quickly, have the ability of self-organization and are high concealment, so they are increasingly popular in a number of application domains such as environment supervision, traffic control and battlefield reconnaissance. In order to supply visual image or video data, video sensor network is widely applied because of the technology development and the cost reduction. Coverage enhancement is a fundamental problem in wireless sensor networks, and many researchers have carried out researches in this area $[1,2,3]$. Most of related researches are based on an omni-directional sensing model. However, video sensor can sense the area within a limited angle only, which is a directional sensor, so traditional research results about the coverage of wireless sensor networks can not be used into wireless video network directly. Thus, coverage enhancement for directional sensor networks has drawn more attention and has become a focus.

Ma et al [4] proposed the concept of model for directional sensing firstly, and explored the integrity and connectivity problems of directional sensing networks. Potential field-based directional sensing coverage enhancing algorithms [5,6,7] have been made a lot of researches. In this kind of methods, each camera is regarded as a virtual particle and can be repelled by neighbor cameras, thus the problem of nodes' direction adjustment is converted into the problem of particles' union distribution, and then the coverage of the directional network can be enhanced effectively through decreasing overlapping and blind area. However, when the forces between nodes are in equilibrium, the directions of nodes are not necessarily the most optimum [8]. But because the directions cannot move anymore, sometimes the coverage rate is affected. In order to overcome the shortage of potential field-based methods, $\mathrm{Xu}$ et al [8][9] proposed to solve the coverage of video sensor network by using PSO. Since traditional PSO is easy to fall into local optima [10], the mentioned coverage methods of video sensor network based on PSO have similar disadvantages such as local optima and low convergence speed.

In this paper, improved PSO algorithm is used in the coverage enhancing of video sensor network to overcome the shortages of traditional methods based on PSO. Experiments show that the proposed method has higher convergence speed and better coverage performance than traditional coverage method based on PSO. 


\section{Coverage-Enhancing for video sensor network based on PSO}

Node model of video sensor network. We adopt node model of video sensor network similar with that in [8]. As illustrated in Figure 1, the position of video sensor node $C$ is $(x, y)$, the angle of field of view is denoted by $2 \alpha$, the azimuth of sensing orientation $\vec{d}$ is defined as $\theta$, and $R$ is the biggest sensing distance of node. All nodes in the video sensor network are deployed randomly over an area. The sensing orientation of node is $360^{\circ}$ adjustable. The fan-shaped area $A B C$ is the sensing field of a node, and the coverage rate of the whole network can be defined as:

$$
\text { coverage_rate }=\frac{\text { Area of being covered region }}{\text { Total area of ROI region }}
$$

where Area of being covered region contain all the areas that covered by one or more nodes.

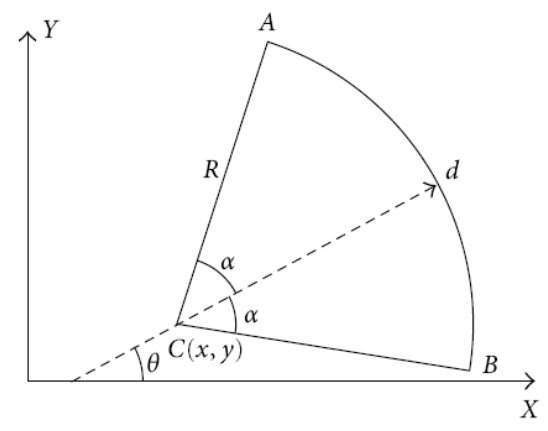

Fig. 1. Node model of video sensor network.

Coverage enhancing method. PSO is an optimization algorithm that can obtain approximate solution for complicated problems. It is effective, simple and easy to implement, and often supplies suboptimal solutions for NP-hard problems. Suppose $x_{i}(t)$ and $v_{i}(t)$ are position and velocity vectors of particle $i$ at generation $t$, then the velocity and position can be updated based on (2) and (3).

$$
\begin{aligned}
& v_{i}(t+1)=w v_{i}(t)+c_{1} r_{1}\left(P_{i}-x_{i}(t)\right)+c_{2} r_{2}\left(G_{\text {best }}-x_{i}(t)\right) \\
& x_{i}(t+1)=x_{i}(t)+v_{i}(t+1)
\end{aligned}
$$

where $w$ means the current velocity of particles are affected by the velocity in the last iteration, so it is often called the inertia factor. $P_{i}$ and $G_{\text {best }}$ store the positions of particle $i$ corresponding to the best solution of local and global optimum respectively during the history of updates. $c_{1}$ means that the velocity is affected by the private best position memorized by the particle. And $c_{2}$ means that the velocity is also affected by the global best position memorized by the system. $c_{1}$ and $c_{2}$ represents the abilities of local extremes and global extremes search respectively. $r_{1}$ and $r_{2}$ are two random numbers in $(0,1), t$ is the generation of particles.

The sensing orientations of nodes were defined as particles in [8], that is $x=\left(\theta_{1}, \theta_{2}, \cdots, \theta_{N}\right)$. The objective function is the total coverage rate defined in (1). The updating process of particle's position is actually the searching and adjusting process of sensor node's orientation. 


\section{Coverage-Enhancing for video sensor network based on improved PSO}

Improved PSO. The inertia factor was constant in traditional PSO algorithm, for example, $w=0.729$ in [8]. A constant inertia means keeping the same degree of attention for local and global search regardless of the iterative processes. It can be seen from (2) that larger inertia weight means more efficient global search of particles, and smaller inertia factor ensures more effective local search. So a new strategy that calculating inertia factor is defined as [10]:

$$
w(t)=w_{\max }-\frac{\left(w_{\max }-w_{\min }\right) \times t}{\operatorname{MaxIT}}
$$

where $w_{\max }$ and $w_{\min }$ are the maximum and minimum values of inertia factor, MaxIT is a predefined maximum iteration time. $w$ is linearly decreases with the increase number of iterations. There are larger $w$ at early of the iteration, which ensure the particles search extremes in the whole possible space with bigger searching step length. And there are smaller at later stage, which make the particles converge quickly to the global optimum position.

Steps of the proposed method. Based on the research and analysis above, we propose to apply the improved PSO algorithm to the coverage enhancement of video sensor network. The implementation steps are as follows:

Step 1: Generate $N$ position vectors randomly.

Step 2: Generate $N$ orientation vectors $x(0)=\left(\theta_{1}, \theta_{2}, \cdots, \theta_{N}\right)$ randomly and regard $x(0)$ as the initial particle, which is the particle for $t=0$.

Step 3: The initial particle is also regarded as the local and global optimum values $P_{i}$ and $G_{b e s t}$. Set $v_{1}(1)=v_{2}(1)=\cdots=v_{N}(1)=v s$ as the initial velocity for each dimension of particle, where $v s$ is a predefined constant.

Step 4: Each dimension of particle is updated based on (3), that is $x_{i}(t+1)=x_{i}(t)+v_{i}(t+1)$, then the new generation particle $x(t+1)$ is obtained.

Step 5: Update $P_{i}$ and $G_{\text {best }}$ based on objective function (1).

Step 6: Calculate inertia for the next generation based on (4), and calculate new velocity for next iteration based on (2).

Step 7: Transfer to step 4 until the PSO reaches its end conditions (the maximum times of iteration or the requirement accuracy of algorithm).

Step 8: Output $G_{\text {best }}$ as the final solution of the algorithm.

\section{Experiments and analysis}

In the experiment, the convergence performance and coverage enhancing effect were investigated. In order to prove the performance of the proposed method, the experimental results were compared to that of the traditional PSO based coverage enhancing algorithm in [8].

Similar experimental environment with that in [8] was adopted for comparison. The interesting objective area was set to be a $500 \times 500$ plane, and 150 nodes were deployed randomly in the area. All nodes had the same parameters, which were $R=50,2 \alpha=90^{\circ}$. The maximum number of loops MaxIT was set to 1000 . Values of some parameters for the proposed algorithm were set as follows: The maximum and minimum values for inertia factor were $w_{\max }=0.9$ and $w_{\min }=0.3$.

Figure 2(a) shows the initial layout effect of the video sensor network, which is deployed randomly. Figure 2(b) is the final layout result of the method in [8] and Figure 2(c) shows the coverage result of the proposed algorithm in this paper. In Figure 2, the covered areas were drawn in gray. It can be seen that the result of the proposed method has less overlapped region, which means higher coverage rate.

The curves of coverage rate to iteration number for two methods are illustrated in Figure 3 . The initial network has coverage rate of 0.5042 . The method in [8] converged after 911 cycles of PSO 
and the coverage was raised to 0.5241 . The proposed method is stable after 312 times iteration and the coverage rate reaches 0.5302 . Our algorithm has higher coverage rate and fast convergence speed.

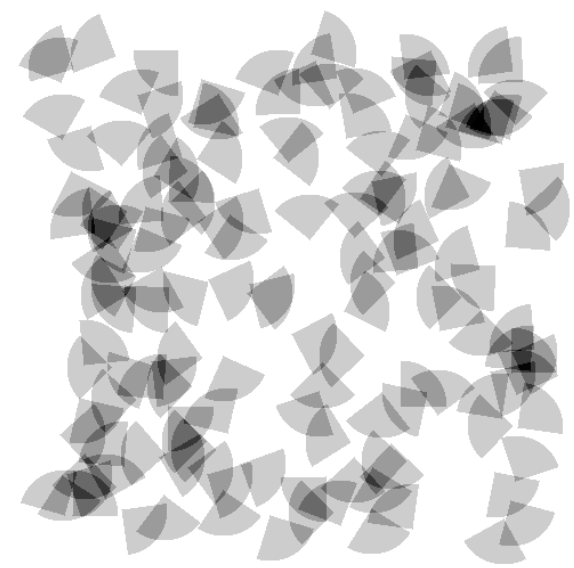

(a) Initial coverage result

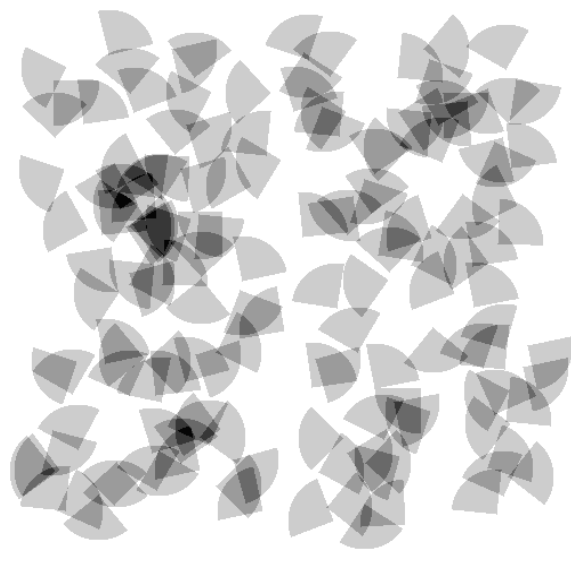

(b) Result for method in [8]

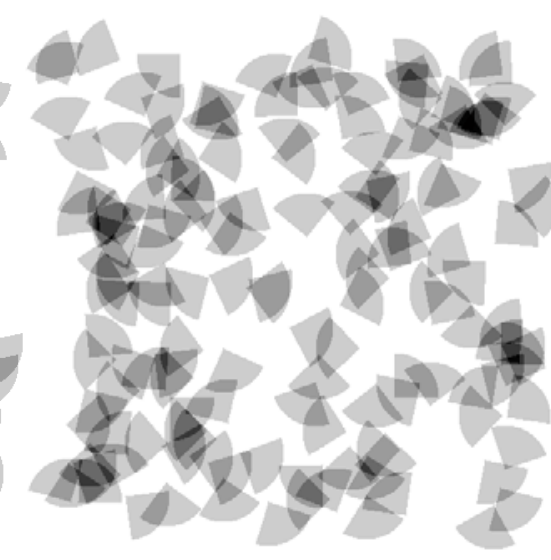

(c)Result for our method

Fig. 2. The coverage results.

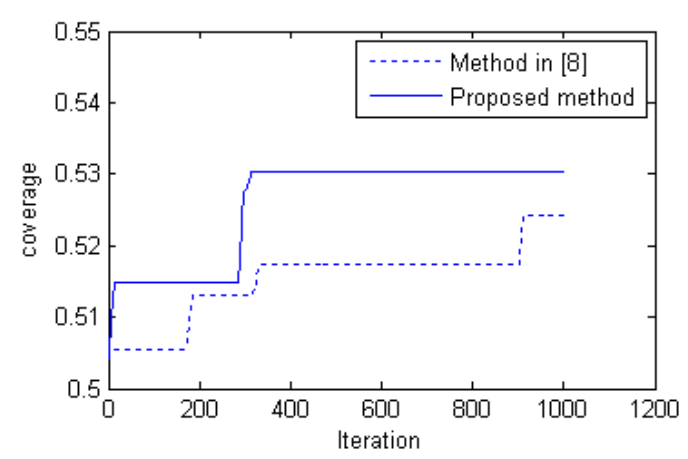

Fig. 3. The curves of iteration number to coverage rate for video sensor network distributed on a $500 \times 500$ area with 150 nodes.

\section{Conclusion}

PSO can converge to the global optimization at a high speed. It is suitable for solving the optimization problem of multi-dimensional functions in continuous space. In this paper, PSO is used to the coverage enhancing of video sensor network. By improving the inertia and learning factors of PSO, the algorithm have high abilities of local searching at the early of iteration, which can prevent it falling into the local optima, and is efficient to global search at the rear phase, which can achieve global optimization.

\section{Acknowledgement}

In this paper, the research was sponsored by the National Natural Science Foundation of China (Project No. 61165011), the Science and Technology Project of Jiangxi Province (Project No. 20122BBF60072) and the Youth Science Foundation of Jiangxi province (Project No. 20132BAB211021).

\section{References}

[1] Tien-Wen Sung, Chu-Sing Yang. Voronoi-based coverage improvement approach for wireless directional sensor networks. Journal of Network and Computer Applications, 201439 (3) 202-213.

[2] Mahmut Karakaya, Hairong Qi. Coverage estimation for crowded targets in visual sensor 
networks. ACM Transactions on Sensor Networks, 20128 (3) 1-22.

[3] Linghe Kong, Mingchen Zhao, Xiaoyang Liu, Jialiang Lu. Surface coverage in sensor networks. IEEE Transaction on Parallel and Distributed Systems, 201425 (1) 234-243.

[4] Ma HD, Liu YH. On Coverage Problems of Directional Sensor Networks . International Conference on Mobile Ad2hoc and Sensor Networks. 2005 721-731.

[5] Li Yupeng, Ji Peng, Liu Hongchao, Jiang Jingqi. A Virtual Potential Field Based Coverage-Enhancing Algorithm for 3D Directional Sensor Networks. The 6th International Conference on New Trends in Information Science and Service Science and Data Mining, 2012 225-230.

[6] Zhao Jing, Zeng Jian-Chao. A virtual potential field based coverage algorithm for directional networks. Control and Decision Conference, 2009 4590-4595.

[7] Dan Tao, Hua-Dong Ma, Liang Liu. Virtual potential field based coverage-enhancing algorithm for directional sensor networks. Journal of Software, 200718 (5) 1152-1163.

[8] Yichun Xu, Bangjun Lei, Emaile A.Hendriks. Camera network coverage improving by particle swarm optimization. Journal on Image and Video Processing, 2011 (3) 1-10.

[9] Xiao Fu, Wang Ruchuan, Sun Lijuan, Wu Shuai. Research on the three-dimensional perception model and coverage-enhancing algorithm for wireless multimedia sensor networks. The Journal of China Universities of Posts and Telecommunications, 2010, 17 (Suppl. 2) 67-72.

[10] Yongshou Dai, Hui Niu. An improved PSO algorithm and its application in seismic wavelet extraction. I. J. Intellligent Systems and Appllications, 20115 34-40. 